\section{THIRD REPORT

\author{
on
}

\section{THE ACTION OF ANÆSTHETICS} To

THE SCIENTIFIC GRANTS COMMITTEE OF THE BRITISH MEDICAL ASSOCIATION.

By a Committee consisting of JOSEPH COATS, M.D., Pathologist to the Western Infirmary, Glasgow; WILLIAM RAMSAY, Ph.D., Assistant to the Professor of Chemistry; and JOHN G. McKENDRICK, M.D., Professor of Physiology in the University of Glasgow.

\section{ON THE EFFECTS OF CHLOROFORM, ETHIDENE, AND ETHER, ON BLOOD-PRESSURE. With a Lithographic Plate.}

THE Committee have been engaged, since their last report, ${ }^{*}$ chiefly in investigating the condition of the blood-pressure in animals under the influence of chloroform, ethidene, and ether.

In the report of a Committeet of the Royal Medical and Chirurgical Society, to inquire into the uses and effects of chloroform, and published in the Transactions for 1874 , vol. xlvii, there is a very excellent but brief record of the blood-pressure under chloroform and ether. The instrument used in these experiments was the hæmadynamometer of Poiseuille, which consists of a U-shaped tube, with mercury in the bend. One limb of the tube was connected with the femoral artery, and the rise of the mercury in the opposite limb indicated the blood-pressure. By means of this instrument, the Committee were enabled to report that, on administering chloroform, there was at first a transient rise of the blood pressure ; after which, there was a gradual, but not a regular, fall. They also noticed that, when the force of the heart was reduced by chloroform to the full extent, the respiration of fresh air was at once followed by a rise of the mercury. In regard to ether, it was found that the primary rise in pressure was greater and more constant than with chloroform, and that the depressing effect was very slight or altogether absent.

In our experiments, we have been able to amplify these results; and, by means of more delicate instruments, to obtain more exact records. We have used a very complete kymograph, in the Physiological Laboratory of the University of Glasgow, made by Rudolph Rothe of Prague, + by means of which the variations in the column of mercury, produced by the pulsations in an artery, are written (by means of a stem which floats on the mercury) on a sheet of blackened paper, which is carried round by a clockwork arrangement. A sheet of paper, eight feet long, is adapted to the machine; and, as three or four lines of tracing can be taken at different levels on the same sheet, a continuous tracing may be obtained of twenty-four, thirty-two, or even more feet. But, further, the sheets can be changed in a few seconds, and so it is possible to take tracings of almost any length.§ The instrument has also appliances by which time could be recorded in seconds, half-seconds, or otherwise, immediately beneath the tracing of the blood-pressure. (See $c$ in plate.) Lastly, there are two arrangements by which the exact time of administering and discontinuing an anæsthetic can be marked. (See $\mathrm{C}$ in plate.) In this way, we have obtained records of experiments in rabbits and dogs, those on dogs being of much the greater value.

In the case of the rabbit, there is one fact of considerable interest which seems to be deducible from these records. When the animal is not fully under chloroform, any fresh administration causes most remarkable variations in blood-pressure, with retardation of the heart's contractions. (See $\mathrm{G}$ and $\mathbf{H}$ in plate.) There is frequently a sudden dip in the pressure, to the extent of forty millimetres out of a total of one hundred and ten millimetres; and in the next few contractions, there is a very rapid rise up to the former level, to be succeeded by another sudden dip. This occurrence followed so uniformly in certain stages of chloroform-narcosis, on every approximation of a sponge containing the agent, even for a few seconds, to the animal's muzzle, that it was regarded as probably reflex. This is rendered all the more probable by the known fact that ammonia-vapour, applied to

* British Medical Journal, January 25 th, 1879.

$\uparrow$ The members of this committee were T. B. Curling, Thomas Bryant, Samuel Cartwright, Arthur Farre, George G. Gascoyen, George Harley, Prescott Hewett, F. W. Mackenzie, William Marcet, Charles H. Moore, James Paget, William O. Priestley, Richard Quain, Francis Sibson, R. Dundas Thomson, Charles West, Septimus W.'Sibley, George W. Callender, John Birkett, and Mr. Clover, assisting.

$\ddagger$ See Dr. McKendrick's Outlines of Physiology, page 358.

$\$$ In one case, a tracing one hundred and fifty feet long was obtained. the nostrils of a rabbit, causes stoppage of the heart's action. ${ }^{*}$ When the chloroform is given continuously, these variations gradually cease, and there succeeds a regular and gradual fall of pressure down to zero, if the agent be pushed. It was noticed that, in one or two instances, ethidene produced sudden variations in pressure similar to those of chloroform, but that ether did not. Our experiments were not fully prosecuted on rabbits in regard to the more permanent effects of these two agents ; but it may be said in general that ether seemed to have no effect on blood-pressure, while ethidene reduced it to a considerable extent, but not to total extinction, like chloroform.

Turning to our experiments on dogs, the very first observation made was an exceedingly striking one. (See $A$ in plate.) The animal used was a black retriever, six or seven months old. Chloroform was given, and during deep anæsthesia a cannula was introduced into the carotid artery, and connected with the kymograph. By the time connection with the kymograph was established, no chloroform had been given for about two minutes. On first making the connection, the pressure registered 104 millimetres, which may be regarded as nearly normal; but now, without any fresh dose, the pressure rapidly fell to zero, with a remark. able retardation of the heart. Each pulsation had a height of 9 millimetres, + and a duration of a second and a half. After this, the pressure rose with remarkable variations, equal to from 13 to 16 millimetres, referable to the respiratory movements. (See right-hand part of Tracing A.) It is to be remembered that the animal had at this time to a great extent recovered from chloroform, as evidenced by the high initial pressure ; and this sudden fall of pressure, is apparently reflex in character; the heart being, perhaps, more liable to such influences under the conditions present. A fresh administration of chloroform at this time led to a fresh variation of pressure somewhat resembling those already referred to in the case of rabbits. On continuing the administration, all irregular variations were abolished, and the pressure gradually fell; but the agent was not in this instance pushed very far. During recovery from this administration, seventy seconds after the chloroform had been removed, and when the pressure had risen to 66 millimetres, there was, without any npparent cause, an occurrence somewhat like that at the outset, but less in degree. (See Tracing $B$ in plate.) The pressure fell to 20 millimitres; and the heart's pulsations became unfrequent, each pulsation taking a second and a half. This continued for six beats, when the pressure rose slowly to 100 millimetres, with recovery of the frequency of the pulsations.

Ether administered to this dog produced little effect on the pressure; if anything, improved it. There was slight diminution of pressure when the animal was struggling and howling.

Ethidene was given while the animal was still to some extent under the influence of ether. The pressure gradually but very slowly fell, and, under repeated doses, reached a minimum of 20 millimetres. The agent being still continued, the pressure began to rise, and had reached 28 millimetres when it was removed. Subsequently, ethidene was again given; and, after prolonged and constant use, the pressure was gradually brought down to 7 millimetres, when the administration was stopped. It should be noted that all this time the respiratory movements were uninterrupted.

The same dog was used for a further set of experiments, which were prosecuted after a short interval, during which the animal partially recovered. $\neq$ The pressure at the beginning of this series registered 1 lo millimetres, and the variations of pressure with respiration were well marked. (See Tracing c. The respiratory curve is seen at the left of the tracing.) Chloroform being given, there was an almost immediate fall of pressure, with considerable variations, and reduction in the frequency of the heart's pulsations. The pressure remained about 70 or 80 millimetres for about half a minute; and then there was a very rapid fall, with great retardation of the pulsations, till the heart almost ceased. There was an interval of three seconds between two of the pulsations, of nine seconds between the next two, and of six seconds between the next; the pressure in these intervals being nil. (See Tracing c.) The chloroform was removed when this rapid fall occurred. During this period, the respiration continued. The pulsations were now resumed; but between each pulsation the pressure was minus, and the pulsation only raised the mercury ro millimetres, generally just to the basementline; while the beats occupied about one second and three-quarters. After twenty-nine of these pulsations, the breathing stopped, while the

* See Professor Rutherford's paper in Joumal of Anatomy and Physiology, vol. vii, p. 283, "Cause of the Retardation of the Pulse which follows Artificial or Voluntary Closure of the Nostrils of the Rabbit.

It will be understood that the figures given represent the column of mercury raised, or the difference in level of the mercury in the two limbs of the U-shaped tube. This will always be double the distance from the point at which the mercury in the
two limbs is level, or from the basement line in the tracing.

$\ddagger$ During the whole of these experiments anæsthesia was complete, and the partial recovery referred to was indicated by a return of blood-pressure to the normal. 
pulsations went on regularly as before. After a few seconds, artificial respiration was used, by alternately compressing the chest and leaving it to expand. This being prosecuted for thirty seconds, spontaneous respiration was resumed, and the pulsations became more marked, having a rather higher excursion than before, but still returning to a pressure of zero between each two pulsations. This continued for about forty-two seconds, when the pressure began to rise; and this went on continuously till, in a hundred and sixty seconds, a height of 100 millimetres was reached.

We have dwelt in some detail on this last experiment, because we regard it as one of unusual interest and importance. It is to be remembered that this animal got chloroform in the usual way, by a cloth saturated with the agent being held over his mouth and nose. $\mathrm{He}$ received no overcose, and the administration only lasted seventy seconds. As bearing on at least one mode of death under chloroform, the relation of the heart's action to respiration is of particular significance. The blood-pressure is enormously reduced, and the pulsations have become so unfrequent as to be virtually ineffectual, yet respiration continues. But respiration stops forty seconds after the heart has resumed, the pulsations being still, however, so ineffectual, that the pressure is even minus. ${ }^{*}$ We believe that the legitimate inference to be drawn is that the stoppage of respiration was not due directly to the chloroform, the inhalation of which had ceased for about forty-eight seconds. It seems likely that the failure of the heart in the first instance, and the insuffciency of its subsequent pulsations, were the cause of the failure of respiration. In such a state of the circulation, the respiratory centres would probably be insufficiently supplied with blood, and be consequently liable to cease acting. In this case, if death had occurred, it would only apparently have been due to the failure of the respiration, the primary failure being that of the heart. To what extent this may apply to human cases we do not venture to speculate.

We now resume consideration of this set of experiments. The animal was allowed to recover considerably, and the pressure had reached 106 millimitres, when ethidene was administered. There was a slow but steady fall of pressure, the lowest point being reached in about I 20 seconds, when the height was 36 millimetres. Continuing the administration, there was a slight rise, up to 50 millimitres, when the administration was discontinued. During the administration, the cardiac pulsations were regular in frequency, with slight variations in the height of the waves, probably depending on respiration.

It will not be necessary to give our further experiments in such detail. In one set, we kept up artificial respiration by means of the pump, + administering the anæsthetics by passing the air through a Wolff's bottle containing the respective agents. In these experiments, chloroform promptly reduced the pressure, which began to recover almost immediately on its removal. On continuous administration, the pressure fell much more gradually than by the ordinary method, and the lowest point reached still represented a considerable pressure, about 65 millimetres. The initial pressure was 132 millimetres. Ethidene was began at a pressure of 80 millimitres, the recovery from the chloroform-depression being incomplete. After prolonged use, there was a fall to 54 millimetres. On removal, a gradual recovery ensued, which attained to 80 millimetres. Ether was then given, when again a slight fall in pressure ensued.

It may here be incidentally remarked that with artificial respiration there were very exaggerated variations of pressure, as shown in tracings $\mathrm{H}$ and $\mathrm{K}$; these respiratory variations, however, only occurred when the animal made respiratory movements coincidently with the pumping. When, as a result of deep anæsthesia, respiration ceased on the part of the animal, the respiratory variations also ceased, although the artificial respiration was kept up.

The next experiment is a somewhat interesting one, offering in a certain sense the converse of one already narrated. The same animal was used as in the last experiment ; the tracheal tube was left in, and the animal breathed through it. The anæsthetics were administered by holding a cloth soaked with the agent over the mouth of the tube. Chloroform was given, and there was an almost immediate fall of pressure, but the fall was gradual, and in 234 seconds had reached 28 millimetres, when the chloroform was stopped. The respiration ceased just after the chloroform had been removed. Artificial respiration was at once resorted to; but in spite of this, the heart ceased beating twentyone seconds afterwards. It is remarked, however, that although there were no indications of the heart's pulsations on the tracing, the pressure was maintained at 28 millimitres, and it is just possible that there may

* That there was no fallacy here was determined by testing the instrument afterwards, when the zero line was found to indicate no pressure.

$\dagger$ The apparatus for artificial respiration used in the Physiological Laboratory, University of Glasgow, is the double-piston pump, made by Rudolph Rothe of Prague, and figured in his price-list. have been slight pulsations too feeble to be recorded. The pause of the heart continued for twenty-one seconds, and the pulsations were then resumed very feebly and irregularly. The pressure rose gradually to 46 millimitres, when a spontaneous respiration was given; then, with long intervals, spontaneous respiration was resumed, and the artificial respiration was stopped, as it was not required.

In this observation, it seems undoubted that the respiration failed first. The rapid failure of the heart is a remarkable circumstance, especially when the comparatively high pressure is considered. It is possible that the introduction of artificial respiration may have had to do with it. The respirations before they ceased were shallow; and though the air in the lungs was saturated with chloroform, little of it would find its way into the blood in the very limited respiratory movements. The introduction of artificial respiration would at first force the saturated air rapidly through the lungs, which would be vigorously inflated, and a large amount of chloroform would be introduced into the blood.

After the animal had recovered from this experiment, ethidene wa given. There was a fall of pressure; but, though administration was continued in frequently repeated doses for nine minutes, the bloodpressure only fell to 38 millimetres, and there was no failure of respiration. Before the ethidene was removed, the pressure had risen to 60 millimitres. (See tracings $\mathrm{D}$ and $\mathrm{E} ; \mathrm{E}$ is continuous with $\mathrm{D}$ after a considerable interval.) The respiratory curve was preserved even at the point of lowest pressure, and the regularity of the heart's pulsations was not interfered with.

In this animal the pneumogastrics were now cut, and the observations were repeated both with and without artificial respiration. It cannot be said that any essential difference was apparent in the results. The pressure fell both with chloroform and with ethidene, but the fall was perhaps not so rapid nor so great as under other circumstances. While artificial respiration was used, ethidene and chloroform were successively pushed to a very great extent, the Wolff's bottle being heated to facilitate the evaporation of the agents. Yet the blood-pressure was not reduced to the lowest, even with this treatment, and in the case of ethidene it even began to rise under it. The effect on the respiratory variations in pressure was remarked during these experiments. In the case of ethidene, even when the agent was given to the fullest extent, the respiratory curve, though very much diminished, did not entirely disappear. In the case of chloroform, the respiratory curve disappeared completely, and that shortly after the commencement of administration. The cardiac pulsations in the case of chloroform became scarcely perceptible, the pressure, however, not falling below 40 millimetres. With ethidene, in the same circumstances, the cardiac pulsations remained of nearly normal amplitude.

As a result of the whole set of experiments with this dog, it may be said that the heart showed throughout a remarkable state of vigour. It only exhibited signs of giving way on one occasion, and in that case the blood-pressure was maintained at a comparatively high position. On the other hand, respiration failed during the administration of chloroform very readily. In these respects, this animal contrasts with the former one. It is quite obvious, also, that on these occasions when the breathing ceased the animal would almost certainly have died, but for the use of artificial respiration.

In some further experiments which we made with another dog, using Fick's kymograph*, a more remarkable result was obtained. 13oth respiration and heart had stopped under the use of chloroform, but by means of artificial respiration (by the pump) there was ultimate recovery, although the pulsations of the heart had ceased for a considerable time. In this case there could not be any feeble pulsations keeping up the circulation at a slow rate, as was supposed to be possible in a former case, because Fick's arrangement registers very accurately the slightest variations in the pressure produced by the heart's action. In the case of this dog also it was observed that several times, after a period of shallow respirations, the breathing stopped for a brief interval, the heart beating with considerable vigour. As respiration hat ceased, no more chloroform was admitted to the blood, and after a time the respiratory movements returned. So much was this the case that diffculty was experienced in killing the dog with chloroform, and this was only effected by administering it by artificial respiration, after the spontaneous movements of respiration had ceased. By artificial respiration an additional quantity was introduced, and the heart soon succumbed.

These facts are of considerable importance, as they show the varying effects of chloroform in the same animal at different times. At one period, respiration and the heart failed nearly at the same time. At another, respiration failed, and the heart, being still vigorous, was able to carry on the circulation till the chloroform had been sufficiently eliminated to allow of the recovery of the respiration.

* The form shown in Dr. McKendrick's Outlines, fig. 93, p. 357. 
The facts obtained from these researches seem to us to warrant the following conclusions.

I. Both chloroform and ethidene administered to animals have a decided effect in reducing the blood-pressure, while ether has no appreciable effect of this kind.

2. Chloroform reduces the pressure much more rapidly and to a greater extent than ethidene.

3. Chloroform has sometimes an unexpected and apparently capricious effect on the heart's action, the pressure being reduced with great rapidity almost to $n i l$, while the pulsations are greatly retarded or even stopped. The occurrence of these sudden and unlooked for effects on the heart's action seems to be a source of serious danger, all the more that in two instances they occurred more than a minute after chloroform had ceased to be administered and after the recovery of the blood-pressure.

4. Ethidene reduces the blood-pressure by regular gradations, and not, so far as observed, by sudden and unexpected depressions.

5. Chloroform may cause death in dogs by primarily paralysing either the heart or the respiration. The variations in this respect seem to depend to some extent on individual peculiarities of the animals; in some, the cardiac centres are more readily affected, in others, the respiratory. But peculiarities in the condition of the same animal very probably have some effect in determining the vulnerability of these two centres respectively, and they may both fail simultaneously.

6. In most cases, respiration stops before the heart'saction; but there was one instancein which respiration continued when the heart had stopped, and only failed a considerable number of seconds after the heart had resumed.

7. The use of artificial respiration was very effective in restoring animals in danger of dying from the influence of chloroform. In one instance, its prolonged use produced recovery, even when the heart had ceased beating for a considerable time.

8. Under the use of ethidene, there was on no single occasion an absolute cessation either of the heart's action or of respiration, although they were sometimes very much reduced. It can, therefore, be said that, though not free from danger on the side of the heart and respiration, this agent is in a very high degree safer than chloroform.

9. These results confirm and amplify those stated in a previous report, to the effect that ethidene does not compromise the heart as does chloroform. By the method of experimentation then employed, the effect on the blood-pressure could not be determined; and altogether the results here obtained are more exact and unequivocal.

It may be added that since the last report ethidene has been given to a number of patients of all ages, with results which may be described as satisfactory. Given freely at first, it produced anæsthesia as rapidly as chloroform, and the effect could readily be kept up by comparatively small subsequent doses. The only drawback is that in some cases it produced vomiting ; but it has not been determined that it does so more frequently than chloroform, over which it has the further advantage of producing less excitement and being more agreeable to the patients.

Isobutyl chloride was given to three patients, but it produced considerable excitement and proved an imperfect anæsthetic.

In concluding this report, the Committee would express the belief that, so far as purely physiological tests go, ethidene has proved itself as efficient an anzesthetic as chloroform, and a much safer one. It is not asserted that this agent ought at once to replace chloroform in practice; but it is believed that a very strong case has been made out for an extensive trial of it. It is impossible for the Committee, having numerous other engagements, to give the agent that extended trial in actual practice which is desirable; and they would ask those who are engaged in practice to use it in their cases, and to report the results they may have obtained.

It appears that ethidene was first employed as an anæsthetic by $\mathrm{Dr}$ Snow. He administered it in fifteen cases, with good results. (See Snow on Chloroform, etc., last paper, published in 1858.) In 1870, it was used by Liebreich and Langenbeck in Berlin. (Berlin. Klin. Wochenschrift, Nos. 31 and 33,1870 , p. 401.) In $187 \mathrm{r}$, two papers appeared ; one by Sauer, in the Pharm. Centralblatt, vol. xiv, p. I40; and the other by Steffen, in Deutsche Klinik, No. 44, p. 398. Sauer mentions one case of death in a patient suffering from heart-disease. In thirty-three cases, two vomited, and two suffered from nausea and headache. In 1872 , Steffen publishes another paper in the same journal, p. 358 , in which he gives details regarding twenty cases; and he states that the results were satisfactory. (See also fahresb. der Mcdicin., I870, I87 I, and I872, where abstracts are given.) Snow states that the difficulty in obtaining ethidene pure may prevent its general use. That difficulty is now removed; and there is little doubt that, if required, ethiclene may be made in a state of purity and at a moderate cost.

Description of Plate.

Some of the tracings referred to in the text are here reproduced, it being understood that in the original the tracings are white on black instead of black on white. The basement line represents the point at which the needle was when the mercury in the two limbs of the manometer was level. According to the pressure, the mercury is pressed down in the one limb and raised in the other, the tracing giving the latter. As the actual pressure equals the weight of the column of mercury raised, this will be represented by the difference in the levels in the two limbs, and will be double the distance from the basement-line to the tracing. The tracings are to be read from left to right.

A. Represents the occurrence mentioned in the text, in which, while a dog was recovering from chloroform, a sudden fall of pressure occurred, with great reduction in the frequency of the heart's contractions. There is a gradual recovery, with striking inequalities of pressure corresponding with respiratory movements.

$B$. Another sudden fall of pressure and retardation of the heart's contractions occurring seventy seconds after the removal of the chloroform, and when the pressure had nearly returned to the normal.

C. The arterial pressure in a dog under the influence of chloroform. There is a progressive but somewhat irregular reduction of pressure, ending in a stoppage of the heart, while respiration continues. There is an imperfect recovery of the heart, the contractions barely causing the pressure to reach the basement line. Beneath this tracing are copies of the markings made with the chronograph, half seconds being registered. The markings used to indicate the administration or leaving off of an agent are also reproduced.

D. The arterial pressure in a dog under the influence of ethidene. There is a regular and gradual reduction in the pressure, the respiratory variations being preserved.

E. This is a continuation of D, after an interval. Ethidene has been given all the time, and the illustration begins at the point where the pressure is lowest. The time, and the illustration begins at the point where the pressure
pressure is now beginning to rise, the ethidene being still continued.

F. The arterial pressure in a dog under the influence of ether. No effect on the F. The arterial pressure in a dog under the influence of ether. No effect
pressure is produced, and the respiratory variations are perfectly preserved. $\mathrm{G}$ and $\mathrm{H}$ show the variations in pressure produced in rabbits by the administration of chloroform, the animals
These are presumed to be reflex.

These are presumed to be reflex. respiration was being carried out. It is to be understood that the animals made synchronous respiratory efforts. The arrangement for artificial respiration not only blew air into the lungs, but sucked it out.

\section{AN ADDRESS \\ INFLUENCE OF POSITION UPON LOCAL CIRCULATION. \\ Read before the Harvcian Socicty of London.*}

By JOSEPH LISTER, F.R.S.,

Professor of Clinical Surgery in King's College, London.

Mr. President and Gentlemen,-Having been honoured by a request from the Council that I would make some communication to this Society, I have thought that no subject could be more appropriately brought before an association connected with the name of Harvey, than some observations begun several years ago, but hitherto unpublished, regarding the effects of the position of a part upon the circulation in it.

My attention was first directed to this subject fifteen years since, when I was engaged in endeavouring to devise a satisfactory method of excising the entire articular apparatus of the wrist for the cure of carious disease. In that operation, although no large arterial branches are divided, the very protracted character of the procedure would render the oozing from small vessels a source of serious loss of blood to the patient if it were allowed to go on unchecked. Accordingly, I was led to deviate from what was then the ordinary practice of restricting the use of the tourniquet to amputation, and employed the instrument in the excision referred to. And I found that, when the hand was raised to the utmost degree, and kept so for a few minutes, and then, while the clevated position was still maintained, a common tourniquet was applied to the arm, being screwed up as rapidly as possible, so as to arrest all circulation in the limb and at the same time avoid venous turgescence, I had a practically bloodless part to operate upon, and thus gained the double advantage of avoiding hæmorrhage and inspecting precisely the parts with which I was dealing. And, having found such great benefit from this bloodless method of operating in the instance referred to, I extended it to other operations on the limbs.

In 1873 , I was one day illustrating this subject to my clinical class in Edinburgh by raising one of my hands to the utmost while the other was kept dependent, in order to exhibit the contrast between them in redness, when a sensation of chilliness coming on in the hand that was raised made me feel, and at once express, the conviction that something more was occurring than could be explained by the mere mechanical effect of the position of the part upon the blood in the vessels, and that the diminution of pressure upon their walls resulting from the action of gravity upon their contained blood must operate as a stimulus to the vaso-motor nervous apparatus of the limb, so as to induce reflex contraction of its arteries. * The paper, as now published, embraces some considerations on which I did not
enter at the time of its delivery, and also some fact subsequently ascertained. 